\title{
Sensorless Adaptive Nonlinear Damping Backstepping Controller for Doubly-Fed Induction Machine in Wind Power Generation
}

\author{
Mohammed Rachidi* and Badr Bououlid Idrissi \\ Université Moulay Ismaïl, Ecole Nationale Supérieure d'Arts et Métiers, BP 4024, \\ Marjane II, Beni Hamed, 50000, Meknès, Morocco \\ *m.rachidi@ensam.umi.ac.ma, i.bououlididrissi@ensam.umi.ac.ma
}

\begin{abstract}
This paper deals with the design of an adaptive controller for Doubly-Fed Induction Generator (DFIG) connected to a stable electrical grid and works in wind generation. The proposed algorithm combines the backstepping technique and a nonlinear damping tool. The aerodynamic torque is supposed to be unknown and will be estimated in real time. In addition, rotor currents of the generator are assumed not to be available for measurements and will be constituted using an observer. This controller achieves the maximum power point tracking (MPPT) and permits to control the reactive power delivered to the grid. The mathematical development of the model and the control design are discussed in detail. The overall stability of the system is shown using Lyapunov theory. Matlab and Simulink based simulation results show the superiority of the proposed controller compared to the conventional controller without adaptation.
\end{abstract}

Keywords: Wind Power Generation, Sensorless control, Adaptive Backstepping, Nonlinear damping, Unknown mechanical torque

\section{Introduction}

Nowadays, the technology using the doubly-fed induction generator (DFIG) as generator is greatly used in the field of wind energy, especially in high power generation. With this machine, the structure usually used is shown in "Figure 1". The stator is directly connected to the three-phase electrical grid, but the rotor is connected via two back-to-back PWM power converters. The capacitor C is used to form the DC voltage source required for the power converters while the coupling filter R-L allows us to connect the grid-side converter (GSC) to the grid. In general, the rotor-side converter (RSC) is used to control the active and reactive power transmitted to the grid while the main function of the grid-side converter (GSC) is to maintain the DC-link voltage constant and to ensure a unity power factor at the output of the R-L filter [1]. Thanks to the reversibility of the converters, the two operating modes (sub-synchronous and super-synchronous) generators are possible [2].

In fact, the main advantage of this structure resides in the reduced dimensioning power of electronic converters, since only 20 to $30 \%$ of the rated power is needed to pass through RSC and GSC converters. This gives a significant reduction in the cost of power electronics components compared to other variable speed generators. Also, this configuration offers a wider range of variable speed which can reach about \pm $30 \%$ around the synchronous speed $[3,4]$. This achieves maximum efficiency of the rotor and allows us to track, continuously, the maximum power point of the wind turbine $[5,6,7]$. 
In the past, control strategies using proportional integral controllers (PI controllers) based on the stator flux or voltage control-oriented approach $[8,9,10]$ were used. But they used to suffer from the system nonlinearities and the problem of uncertainties which affect the robustness of the controllers. Then, nonlinear control is used to solve this question [11] to [17].

The objective of this paper is to take a part in this research area through an Adaptive Backstepping Controller based on a nonlinear damping tool. The proposed controller ensures speed and current tracking objective for a DFIG-based system connected to a stable electrical grid and works in wind generation. In this study, we focused on a very realistic situation in which the aerodynamic torque applied to the wind turbine is assumed to be unknown and the rotor currents are not accessible for measurement. In fact, an accurate modeling of the aerodynamic torque is not an easy task, especially for a very fluctuating wind. In addition, the presence of the slip rings-brushes system for the wound induction machine makes it difficult to measure the rotor currents. For this reason, the proposed algorithm allows us to update the aerodynamic torque in real time and at the same time to estimate the rotor currents.

The paper is structured as follows: The second section presents the model of the global system followed by the control strategy. In the third section, the mathematical development of our nonlinear controller dealt with in detail for both RSC and GSC converters. In the last part, some simulation results are presented to show the effectiveness and validity of the proposed control.

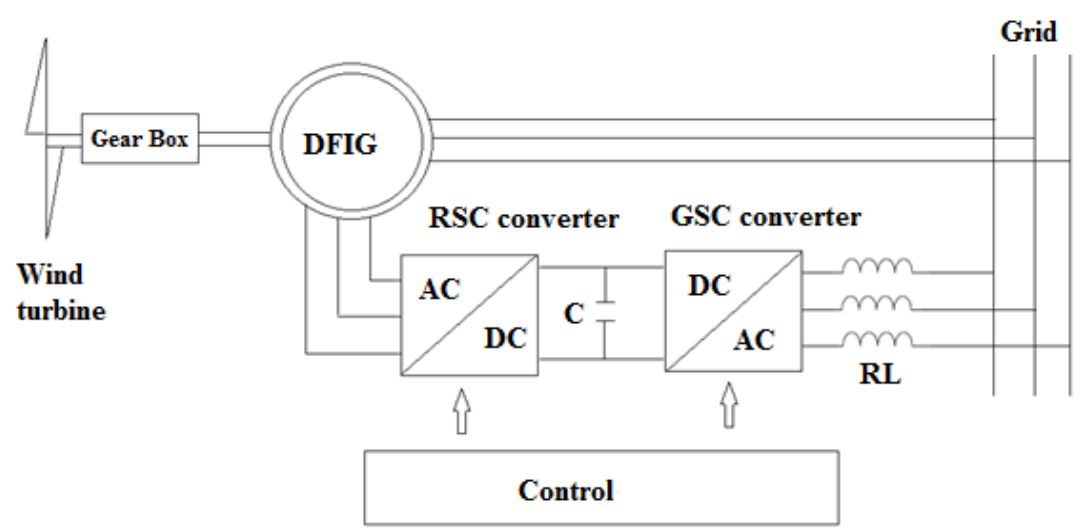

Figure 1. Wind Energy Conversion System

\section{System Model and Control Strategy}

\subsection{Modeling of the Wind Turbine}

The aerodynamic turbine power $\mathrm{P}_{\mathrm{t}}$ depends on the power coefficient $\mathrm{Cp}$ as follows [15]: $P_{t}=\frac{1}{2} \rho \pi R_{t}^{2} C_{p}(\lambda, \beta) v^{3}$

Where $\rho, \mathrm{v}, \mathrm{Rt}, \mathrm{Cp}, \beta, \Omega$ et $\lambda$ are the specific mass of the air, the wind speed, the radius of the turbine, the power coefficient, the blade pitch angle, the generator speed and the Tip Speed Ratio (TSR), respectively.

The TSR is given by: $\quad \lambda=\frac{\mathrm{R}_{\mathrm{t}} \Omega}{\mathrm{G} \mathrm{v}}$

Where: $\mathrm{G}$ is the speed multiplier ratio.

The following equation is used to express $\operatorname{cp}(\lambda, \beta)[15]$ as: 


$$
\mathrm{C}_{\mathrm{p}}(\lambda, \beta)=\mathrm{c}_{1}\left(\frac{\mathrm{c}_{2}}{\lambda_{\mathrm{i}}}-\mathrm{c}_{3} \beta-\mathrm{c}_{4}\right) \exp \left(-\frac{\mathrm{c}_{5}}{\lambda_{\mathrm{i}}}\right)+\mathrm{c}_{6} \lambda
$$

Where $\frac{1}{\lambda_{i}}=\frac{1}{\lambda+0.08 \beta}-\frac{0.035}{\beta^{3}+1}$ and $c_{1}$ to $c_{6}$ are constants coefficients given in

"Appendix 1". The power coefficient reaches its maximum $\left(\mathrm{c}_{\mathrm{pmax}}\right)$ for $\beta=0^{\circ}$ and a particular value $\lambda_{\text {opt }}$ of $\lambda$. In this paper, we suppose that the wind turbine operates with $\beta=0$. To extract the maximum power and hence keep the TSR at $\lambda_{\text {opt }}$ (MPPT strategy), a speed control must be done. According to (1b), the optimal mechanical speed is:

$$
\Omega_{\mathrm{c}}=\frac{\mathrm{Gv}}{\mathrm{R}_{\mathrm{t}} \lambda_{\mathrm{opt}}}
$$

\subsection{Induction Generator Modeling}

To get the model of the induction machine, we have applied the Park transformation in the synchronously rotating frame, for which the $\mathrm{d}$-axis is oriented along the stator-voltage vector position $\left(\mathrm{v}_{\mathrm{sd}}=\mathrm{V}, \mathrm{v}_{\mathrm{sq}}=0\right)$. In this reference frame, the electromechanical equations are [16]:

$$
\begin{aligned}
& {[\mathrm{V}]=[\mathrm{R}][\mathrm{I}]+\frac{\mathrm{d}[\phi]}{\mathrm{dt}}+[\omega][\phi]} \\
& {[\phi]=[\mathrm{M}][\mathrm{I}]} \\
& \mathrm{J} \frac{\mathrm{d} \Omega}{\mathrm{dt}}=\mathrm{T}_{\mathrm{t}}-\mathrm{T}_{\mathrm{em}}-\mathrm{f} \Omega \\
& \mathrm{T}_{\mathrm{em}}=\mathrm{pL}_{\mathrm{m}}\left(\mathrm{i}_{\mathrm{sq}} \mathrm{i}_{\mathrm{rd}}-\mathrm{i}_{\mathrm{sd}} \mathrm{i}_{\mathrm{rq}}\right)
\end{aligned}
$$

Where $[R]=\left[\begin{array}{cccc}\mathrm{Rs} & 0 & 0 & 0 \\ 0 & \mathrm{Rs} & 0 & 0 \\ 0 & 0 & \mathrm{Rr} & 0 \\ 0 & 0 & 0 & \mathrm{Rr}\end{array}\right] ; \quad[\mathrm{M}]=\left[\begin{array}{cccc}\mathrm{Ls} & 0 & \mathrm{~L}_{\mathrm{m}} & 0 \\ 0 & \mathrm{Ls} & 0 & \mathrm{~L}_{\mathrm{m}} \\ \mathrm{L}_{\mathrm{m}} & 0 & \mathrm{Lr} & 0 \\ 0 & \mathrm{~L}_{\mathrm{m}} & 0 & \mathrm{Lr}\end{array}\right] ; \quad[\omega]=\left[\begin{array}{cccc}0 & -\omega_{\mathrm{s}} & 0 & 0 \\ \omega_{\mathrm{s}} & 0 & 0 & 0 \\ 0 & 0 & 0 & -\omega_{\mathrm{r}} \\ 0 & 0 & \omega_{\mathrm{r}} & 0\end{array}\right]$

$[V],[I]$ and $[\Phi]$ are the voltage, the current and the flux vectors. The subscripts s, $\mathrm{r}, \mathrm{d}$ and $\mathrm{q}$ stand for stator, rotor, direct and quadratic, respectively.

$\mathrm{R}, \mathrm{L}, \mathrm{L}_{\mathrm{m}}, \omega, \mathrm{J}, \mathrm{f}, \mathrm{p}, \mathrm{T}_{\mathrm{t}}$ and $\mathrm{T}_{\mathrm{em}}$ are resistance, inductance, mutual inductance, electrical speed, total inertia, damping coefficient, number of pole pairs, mechanical torque and electromagnetic torque, respectively.

By choosing currents and speed as state variables, the system (2) can be put into the following state-space form:

$$
\begin{aligned}
& \dot{\mathrm{x}}_{1}=\mathrm{g}_{1}(\mathrm{x})+\beta \mathrm{u}_{1} \\
& \dot{\mathrm{x}}_{2}=\mathrm{g}_{2}(\mathrm{x})+\beta \mathrm{u}_{2} \\
& \dot{\mathrm{x}}_{3}=\mathrm{g}_{3}(\mathrm{x})+\alpha \mathrm{u}_{1} \\
& \dot{\mathrm{x}}_{4}=\mathrm{g}_{4}(\mathrm{x})+\alpha \mathrm{u}_{2} \\
& \dot{\eta}=-\mathrm{F} \eta+\Gamma_{\mathrm{t}}+\Gamma_{\mathrm{em}}=-\mathrm{F} \eta+\Gamma_{\mathrm{t}}+\mathrm{h}(\mathrm{x})
\end{aligned}
$$

Where

$$
\begin{aligned}
& \mathrm{x}=\left(\mathrm{x}_{1}, \mathrm{x}_{2}, \mathrm{x}_{3}, \mathrm{x}_{4}\right)^{\mathrm{t}}=\left(\mathrm{i}_{\mathrm{sd}}, \mathrm{i}_{\mathrm{sq}}, \mathrm{i}_{\mathrm{rd}}, \mathrm{i}_{\mathrm{rq}}\right)^{\mathrm{t}}, \eta=\Omega \\
& \mathrm{h}(\mathrm{x})=\Gamma_{\mathrm{em}}=\frac{-\mathrm{T}_{\mathrm{em}}}{\mathrm{J}}=\mathrm{a}\left(\mathrm{x}_{1} \mathrm{x}_{4}-\mathrm{x}_{2} \mathrm{x}_{3}\right), \mathrm{u}_{1}=\mathrm{v}_{\mathrm{rd}}, \mathrm{u}_{2}=\mathrm{v}_{\mathrm{rq}}, \quad \alpha=\frac{1}{\sigma \mathrm{L}_{\mathrm{r}}}, \beta=\frac{-\mathrm{L}_{\mathrm{m}}}{\sigma \mathrm{L}_{\mathrm{r}} \mathrm{L}_{\mathrm{s}}}
\end{aligned}
$$




$$
\begin{aligned}
\quad g_{1}(x)=-a_{1} x_{1}+b_{1} x_{2}+c_{1} x_{3}+m_{1} x_{2} \eta+n_{1} x_{4} \eta+\alpha_{u} ; g_{2}(x)=-b_{1} x_{1}-a_{1} x_{2}+c_{1} x_{4}-m_{1} x_{1} \eta-n_{1} x_{3} \eta \\
\quad g_{3}(x)=c_{3} x_{1}-a_{3} x_{3}+b_{3} x_{4}-n_{3} x_{2} \eta-m_{3} x_{4} \eta+\beta_{u} ; g_{4}(x)=c_{3} x_{2}-b_{3} x_{3}-a_{3} x_{4}+n_{3} x_{1} \eta+m_{3} x_{3} \eta \\
\mathrm{a}=\frac{p L_{m}}{J}, a_{1}=\frac{R_{s}}{\sigma L_{s}}, b_{1}=\omega_{s}=2 \pi f_{r}, c_{1}=\frac{L_{m} R_{r}}{\sigma L_{s} L_{r}}, m_{1}=p \frac{1-\sigma}{\sigma}, n_{1}=p \frac{L_{m}}{\sigma L_{s}}, \alpha_{u}=\frac{1}{\sigma L_{s}} V, \\
a_{3}=\frac{R_{r}}{\sigma L_{r}}, b_{3}=2 \pi f_{r}, c_{3}=\frac{L_{m} R_{s}}{\sigma L_{s} L_{r}}, m_{3}=\frac{p}{\sigma}, n_{3}=p \frac{L_{m}}{\sigma L_{r}}, \beta=\beta V ; \quad \sigma=1-\frac{L_{m}^{2}}{L_{s} L_{r}} ; F=\frac{f}{J} ; \Gamma_{t}=\frac{T_{t}}{J}
\end{aligned}
$$

$\mathrm{f}_{\mathrm{r}}$ and $\mathrm{V}$ are the constant frequency and magnitude of grid voltage, respectively. Equations (3a) to (3d) can be put into the following compact form:

$$
\dot{\mathrm{x}}=\mathrm{g}(\mathrm{x})+\mathrm{Au}
$$

Where $g(x)=\left(g_{1}(x), g_{2}(x), g_{3}(x), g_{4}(x)\right)^{t}, u=\left(u_{1}, u_{2}\right)^{t}, A=\left[\begin{array}{cccc}\beta & 0 & \alpha & 0 \\ 0 & \beta & 0 & \alpha\end{array}\right]^{t}$

\subsection{DC-link and Coupling Filter R-L Models}

The GSC converter is connected to the grid through an R-L filter "Figure 2" [16].

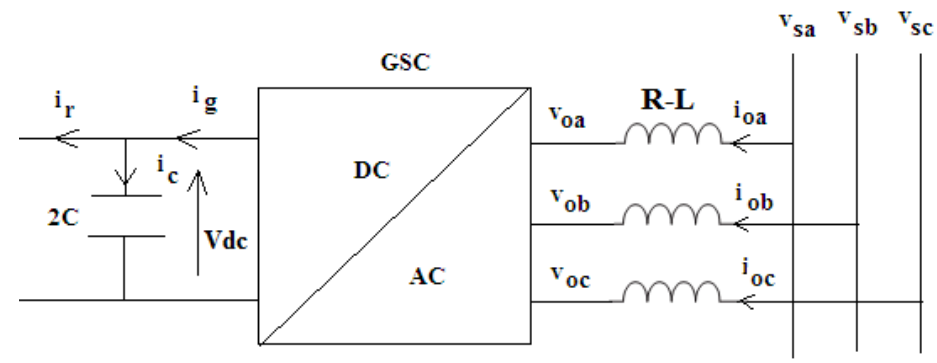

Figure 2. DC-link and R-L Filter Sign Conventions

By using Kirchhoff's laws and Park transformation in the previous rotating frame $\left(\mathrm{v}_{\mathrm{sd}}=\mathrm{V}, \mathrm{v}_{\mathrm{sq}}=0\right)$, we obtain the R-L filter as:

$$
\left\{\begin{array}{l}
\mathrm{v}_{\mathrm{sd}}=\mathrm{Ri}_{0 \mathrm{~d}}+\mathrm{L} \frac{\mathrm{di}}{\mathrm{dt}}-\omega_{\mathrm{s}} \mathrm{i}_{0 \mathrm{q}}+\mathrm{v}_{0 \mathrm{~d}}=\mathrm{V} \\
\mathrm{v}_{\mathrm{sq}}=\mathrm{Ri}_{0 \mathrm{q}}+\mathrm{L} \frac{\mathrm{di}}{\mathrm{dt}}+\omega_{\mathrm{s}} \mathrm{i}_{0 \mathrm{~d}}+\mathrm{v}_{0 \mathrm{q}}=0
\end{array}\right.
$$

Neglecting losses, the DC-link equations are:

$$
\left\{\begin{array}{l}
2 C \frac{d V_{d c}}{d t}=i_{g}-i_{r} \\
v_{s d} i_{0 d}+v_{s q} i_{0 q}=V_{d c} i_{g} \\
u_{1} x_{3}+u_{2} x_{4}=V_{d c} i_{r}
\end{array}\right.
$$

Equations (4) and (5) can be put into the following state-space form:

$$
\begin{aligned}
& \dot{\zeta}_{1}=-b \zeta_{1}+c \zeta_{2}-\mu+\lambda v_{1}=f_{1}(\zeta)+\lambda v_{1} \\
& \dot{\zeta}_{2}=-c \zeta_{1}-b \zeta_{2}+\lambda v_{2}=f_{2}(\zeta)+\lambda v_{2} \\
& \dot{\zeta}_{3}=\alpha(x, u)+\gamma \zeta_{1}
\end{aligned}
$$

Where $\zeta_{1}=\mathrm{i}_{\text {od }} ; \zeta_{2}=\mathrm{i}_{\text {oq }} ; \zeta_{3}=\mathrm{v}_{\mathrm{dc}}^{2} ; \mathrm{v}_{1}=\mathrm{v}_{\mathrm{od}} ; \mathrm{v}_{2}=\mathrm{v}_{\mathrm{oq}}$;

$\mathrm{b}=\frac{\mathrm{R}}{\mathrm{L}}, \quad \mathrm{c}=\frac{\omega_{\mathrm{s}}}{\mathrm{L}}, \quad \mu=-\frac{\mathrm{V}}{\mathrm{L}}, \quad \lambda=-\frac{1}{\mathrm{~L}} \alpha(\mathrm{x}, \mathrm{u})=-\frac{1}{\mathrm{C}}\left(\mathrm{u}_{1} \mathrm{x}_{3}+\mathrm{u}_{2} \mathrm{x}_{4}\right), \quad \gamma=\frac{\mathrm{V}}{\mathrm{C}}$ 


\subsection{Backstepping Control Strategy}

The RSC converter ensures a decoupled control of the stator reactive power and the rotor speed: Indeed, the following expression of the stator reactive power [16]

$$
\mathrm{Q}_{\mathrm{sg}}=\operatorname{Im}\left(\underline{\mathrm{V}}^{*}\right)=\mathrm{v}_{\mathrm{sq}} \mathrm{i}_{\mathrm{sd}}-\mathrm{v}_{\mathrm{sd}} \mathrm{i}_{\mathrm{sq}}=-\mathrm{V} \mathrm{x}_{2}
$$

shows that the current variable $\mathrm{x} 2$ can be exploited to control $\mathrm{Q}_{\mathrm{sg}}$. According to equation (3b), it is clear that the control variable $\mathrm{u}_{2}$ can be designed so that the current $x_{2}$ can track its reference. On the other hand, Equation (3e) shows that $\Gamma_{\mathrm{em}}$ can be regarded as a virtual control for the speed $\eta$. By using the following dynamic equation obtained from (3)

$\dot{\Gamma}_{\mathrm{em}}=\nabla \mathrm{h} \cdot \mathrm{g}+\mathrm{a}\left(\beta \mathrm{x}_{4}-\alpha \mathrm{x}_{2}\right) \mathrm{u}_{1}+\mathrm{a}\left(\alpha \mathrm{x}_{1}-\beta \mathrm{x}_{3}\right) \mathrm{u}_{2}$

the control law $\mathrm{u}_{1}$ can be designed so that the virtual control $\Gamma_{\mathrm{em}}$ can track its reference.

For the GSC converter, the two control variables $\mathrm{v}_{1}$ and $\mathrm{v}_{2}$ allow us to set two control objectives for the GSC system:

- DC-link regulation: with notations used in (6), the variable $\zeta_{3}$ must track a constant reference. From (6c), we can consider $\zeta_{1}$ as a virtual control for the variable $\zeta_{3}$. The Equation (6a) allows us to have access to the control law $v_{1}$.

- Control of the reactive power injected in the grid by the rotor which is given by:

$$
\mathrm{Q}_{\mathrm{r}}=\operatorname{Im}\left(\underline{\mathrm{V}}^{\mathrm{*}}\right)=\mathrm{v}_{\mathrm{sq}} \mathrm{i}_{\text {od }}-\mathrm{v}_{\mathrm{sd}} \mathrm{i}_{\text {oq }}=-\mathrm{V} \zeta_{2}
$$

The Equation (6b) can be used to design the control law $\mathrm{v}_{2}$, so that $\zeta_{2}$ can track its reference.

\section{Control Design}

\subsection{RSC Control}

Our study focuses on the case where the aerodynamic torque is unknown and slowly varied in time. We also assume that the rotor currents $\mathrm{x}_{3}$ and $\mathrm{x}_{4}$ are not available for measurements.

One defines error variables as:

$$
\tilde{\mathrm{x}}_{3}=\mathrm{x}_{3}-\hat{\mathrm{x}}_{3}, \quad \tilde{\mathrm{x}}_{4}=\mathrm{x}_{4}-\hat{\mathrm{x}}_{4}, \quad \tilde{\Gamma}_{\mathrm{t}}=\Gamma_{\mathrm{t}}-\hat{\Gamma}
$$

Where $\hat{x}_{3}, \hat{x}_{4}$ and $\hat{\Gamma}$ denote the observed currents $\mathrm{x}_{3}, \mathrm{x}_{4}$ and the estimated value of $\Gamma_{\mathrm{t}}$, respectively.

In this subsection, we propose a rotor current observer and show its convergence. Then, the nonlinear damping system with unknown nonlinear disturbances and the control design are presented.

\section{1. 1. Rotor Current Observer and Nonlinear Damping System Formulation}

A simple current observer for $\mathrm{x}_{3}$ and $\mathrm{x}_{4}$ is constructed as a copy of the Equations $(3 \mathrm{c})$ and $(3 \mathrm{~d})$ :

$$
\left\{\begin{array}{l}
\dot{\hat{\mathrm{x}}}_{3}=\mathrm{g}_{3}(\hat{\mathrm{x}})+\alpha \mathrm{u}_{1} \\
\dot{\hat{\mathrm{x}}}_{4}=\mathrm{g}_{4}(\hat{\mathrm{x}})+\alpha \mathrm{u}_{2}
\end{array}\right.
$$

Where: $\hat{x}=\left(x_{1}, x_{2}, \hat{x}_{3}, \hat{x}_{4}, \eta\right)^{t}$

Subtracting (11) from ( $3 \mathrm{c}$ and $3 \mathrm{~d}$ ) and using (10), the error equations are given as: 


$$
\left\{\begin{array}{l}
\dot{\tilde{\mathrm{x}}}_{3}=-\mathrm{a}_{3} \tilde{\mathrm{x}}_{3}+\left(\mathrm{b}_{3}-\mathrm{m}_{3} \eta\right) \tilde{\mathrm{x}}_{4} \\
\dot{\tilde{\mathrm{x}}}_{4}=\left(-\mathrm{b}_{3}+\mathrm{m}_{3} \eta\right) \tilde{\mathrm{x}}_{3}-\mathrm{a}_{3} \tilde{\mathrm{x}}_{4}
\end{array}\right.
$$

This second-order system is globally exponentially stable. This can be established using the following Lyapunov function:

$$
\mathrm{V}_{\mathrm{obs}}=\frac{1}{2}\left(\tilde{\mathrm{x}}_{3}^{2}+\tilde{\mathrm{x}}_{4}^{2}\right)
$$

Since its derivative along the solutions of (12) is negative:

$\dot{\mathrm{V}}_{\mathrm{obs}}=-\mathrm{a}_{3}\left(\tilde{\mathrm{x}}_{3}^{2}+\tilde{\mathrm{x}}_{4}^{2}\right) \leq 0$

With this convergent observer and using (10), one can rewrite the RSC system Equations (3) as:

$$
\begin{aligned}
& \dot{\mathrm{x}}_{1}=\mathrm{g}_{1}(\hat{\mathrm{x}})+\varphi_{1}^{\mathrm{t}} \Delta+\beta \mathrm{u}_{1} \\
& \dot{\mathrm{x}}_{2}=\mathrm{g}_{2}(\hat{\mathrm{x}})+\varphi_{2}^{\mathrm{t}} \Delta+\beta \mathrm{u}_{2} \\
& \dot{\hat{\mathrm{x}}}_{3}=\mathrm{g}_{3}(\hat{\mathrm{x}})+\alpha \mathrm{u}_{1} \\
& \dot{\hat{\mathrm{x}}}_{4}=\mathrm{g}_{4}(\hat{\mathrm{x}})+\alpha \mathrm{u}_{2} \\
& \dot{\eta}=\hat{\mathrm{g}}_{5}(\eta)+\varphi_{0}^{\mathrm{t}} \Delta+\hat{\Gamma}_{\mathrm{em}}
\end{aligned}
$$

Where: $\left\{\begin{array}{l}\Delta=\left(\tilde{\mathrm{x}}_{3}, \tilde{\mathrm{x}}_{4}, \tilde{\Gamma}_{\mathrm{t}}\right)^{\mathrm{t}} ; \varphi_{0}^{\mathrm{t}}=\left(-\mathrm{ax}_{2}, \mathrm{ax}_{1}, 1\right) ; \varphi_{1}^{\mathrm{t}}=\left(\mathrm{c}_{1}, \mathrm{n}_{1} \eta, 0\right) ; \varphi_{2}^{\mathrm{t}}=\left(-\mathrm{n}_{1} \eta, \mathrm{c}_{1}, 0\right) \\ \hat{\mathrm{g}}_{5}(\eta)=-\mathrm{F} \eta+\hat{\Gamma} ; \hat{\Gamma}_{\mathrm{em}}=\mathrm{a}\left(\mathrm{x}_{1} \hat{\mathrm{x}}_{4}-\mathrm{x}_{2} \hat{\mathrm{x}}_{3}\right)=\mathrm{h}(\hat{\mathrm{x}}) \\ \hat{\mathrm{x}}=\left(\mathrm{x}_{1}, \mathrm{x}_{2}, \hat{\mathrm{x}}_{3}, \hat{\mathrm{x}}_{4}, \eta\right)^{\mathrm{t}}=\left(\mathrm{i}_{\mathrm{sd}}, \mathrm{i}_{\mathrm{sq}}, \hat{\mathrm{i}}_{\mathrm{rd}}, \hat{\mathrm{i}}_{\mathrm{rq}}, \Omega\right)^{\mathrm{t}} \text { and }\left(\mathrm{u}_{1}, \mathrm{u}_{2}\right)=\left(\mathrm{v}_{\mathrm{rd}}, \mathrm{v}_{\mathrm{rq}}\right)\end{array}\right.$

In the equations system (14), the $\Delta$-terms contain unknown disturbances. To control the system in the presence of such uncertainty, we have combined the backstepping technique $[18,19]$ with a nonlinear damping lemma given in “Appendix 2" [18].

\subsubsection{Control and Update Laws}

One denotes $\eta_{\mathrm{c}}, \Gamma_{\mathrm{emc}}$ and $\mathrm{x}_{2 \mathrm{c}}$ the references for the controlled variables $\eta, \hat{\Gamma}_{\mathrm{em}}$ and $x_{2}$. The next proposition gives the control laws $\mathbf{u}_{1}, \mathbf{u}_{2}$ and the update law $\hat{\Gamma}$.

Proposition 1: The following control and update laws:

$$
\mathrm{u}_{2}=\frac{-\mathrm{g}_{2}(\hat{\mathrm{x}})-\mathrm{k}_{0} \mathrm{z}_{0}-\mathrm{d}_{0} \mathrm{z}_{0}\left\|\varphi_{2}\right\|^{2}+\dot{\mathrm{x}}_{2 \mathrm{c}}}{\beta}, \quad \mathrm{u}_{1}=\frac{-\mathrm{k}_{2} \mathrm{z}_{2}-\mathrm{z}_{1}-\psi_{21}-\mathrm{d}_{2} \mathrm{z}_{2}\left\|\psi_{22}\right\|^{2}-\mathrm{a}_{22} \mathrm{u}_{2}}{\mathrm{a}_{21}}
$$

$\left\{\begin{array}{l}\dot{\xi}=-\lambda_{\mathrm{t}} \xi-\lambda_{\mathrm{t}} \mathrm{h}(\hat{\mathrm{x}})+\lambda_{\mathrm{t}}\left(\mathrm{F}-\lambda_{\mathrm{t}}\right) \eta-\mathrm{v}_{\mathrm{t}} \\ \hat{\Gamma}_{\mathrm{t}}=\xi+\lambda_{\mathrm{t}} \eta \\ \text { Where }: \mathrm{v}_{\mathrm{t}}=-\mathrm{z}_{1}-\mathrm{z}_{2}\left(\mathrm{f}_{6}+\lambda_{\mathrm{t}}\right)\end{array}\right.$

Associated to the following observer:

$\dot{\hat{\mathrm{x}}}_{3}=\mathrm{g}_{3}(\hat{\mathrm{x}})+\alpha \mathrm{u}_{1}$

$\dot{\hat{\mathrm{x}}}_{4}=\mathrm{g}_{4}(\hat{\mathrm{x}})+\alpha \mathrm{u}_{2}$ 
With

$$
\begin{aligned}
& z_{0}=x_{2}-x_{2 c} ; z_{1}=\eta-\eta_{c} ; z_{2}=\hat{\Gamma}_{e m}-\Gamma_{e m c} ; \quad \hat{\Gamma}_{e m}=h(\hat{x}) ; \\
& \Gamma_{e m c}=-k_{1} z_{1}-g_{5}(\hat{x})+\dot{\eta}_{C}-d_{1} z_{1}\left\|\varphi_{0}\right\|^{2} ; \varphi_{0}^{t}=\left(-a x_{2}, a x_{1}, 1\right) ; \\
& \varphi_{2}^{t}=\left(-n_{1} \eta, c_{1}, 0\right) ;\left(\psi_{22}\right)^{t}=\left(f_{1} c_{1}-f_{2} n_{1} \eta-f_{6} a x_{2} ; f_{1} n_{1} \eta+f_{2} c_{1}+f_{6} a x_{1} ; f_{6}+\lambda_{t}\right) \\
& \psi_{21}=f_{1} g_{1}(\hat{x})+f_{2} g_{2}(\hat{x})+f_{3} g_{3}(\hat{x})+f_{4} g_{4}(\hat{x})+f_{5} \dot{\eta}_{C}+f_{6}\left(\hat{g}_{5}(\eta)+\hat{\Gamma}_{e m}-\dot{\eta}_{C}\right)-\ddot{\eta}_{C}-v_{t} \\
& a_{21}=\beta f_{1}+\alpha f_{3} ; \quad a_{22}=\beta f_{2}+\alpha f_{4} ; \quad f_{1}=a \hat{x}_{4}+2 d_{1} z_{1} a^{2} x_{1} ; \quad f_{2}=-a \hat{x}_{3}+2 d_{1} z_{1} a^{2} x_{2} ; \\
& f_{3}=-a x_{2} ; \quad f_{4}=a x_{1} ; \quad f_{5}=-\hat{F} ; f_{6}=k_{1}-\hat{F}+d_{1}\left(a^{2} x_{1}^{2}+a^{2} x_{2}^{2}+1\right) \\
& k_{0}, k_{1}, k_{2}, \lambda_{t}, d_{0}, d_{1}, d_{2} \text { are positives design constants }
\end{aligned}
$$

ensure the asymptotic stability for the RSC system.

\section{Proof:}

\section{a- Control law $u_{2}$}

As mentioned before, the variable $\mathrm{u}_{2}$ can be used to control the current $\mathrm{x}_{2}$. We will first determine the dynamic equation of the error variable $z_{0}$ which is defined as:

$\mathrm{z}_{0}=\mathrm{x}_{2}-\mathrm{x}_{2 \mathrm{c}}$

Using (14b), we can write:

$$
\dot{\mathrm{z}}_{0}=v+\varphi_{2}^{\mathrm{t}} \Delta
$$

Where $v$ is an intermediate control variable given by:

$\mathrm{v}=\mathrm{g}_{2}(\hat{\mathrm{x}})+\beta \mathrm{u}_{2}-\dot{\mathrm{x}}_{2 \mathrm{c}}$

If the $\Delta$-term is not present $\dot{\mathrm{z}}_{0}$ becomes

$\dot{\mathrm{z}}_{0}=\mathrm{v}$

Using $\mathrm{V}_{0}=\frac{1}{2} \mathrm{z}_{0}^{2}$, as a Lyapunov function candidate, and choosing $\dot{\mathrm{z}}_{0}=-\mathrm{k}_{0} \mathrm{z}_{0}$, where $\mathrm{k}_{0}$ is a positive design constant, yields $\dot{\mathrm{V}}_{0}=-\mathrm{k}_{0} \mathrm{z}_{0}^{2} \leq 0$.

Hence, the law $v$ that stabilizes the unperturbed system verifies:

$$
\mathrm{v}=-\mathrm{k}_{0} \mathrm{z}_{0}
$$

To compensate for the presence of $\Delta$-term, the nonlinear damping lemma is used and gives $\mathrm{v}=-\mathrm{k}_{0} \mathrm{z}_{0}-\mathrm{d}_{0} \mathrm{z}_{0}\left\|\varphi_{2}\right\|^{2}$

In fact, the lemma is applied to the system (16) where: $\mathrm{x}=\mathrm{z}_{0}, \mathrm{~V}=\mathrm{V}_{0}, \varphi=\varphi_{2}$ and $\mathrm{g}(\mathrm{x})=1$

Hence, Eq (17) gives the control law $u_{2}$ as:

$$
\mathrm{u}_{2}=\frac{-\mathrm{g}_{2}(\hat{\mathrm{x}})-\mathrm{k}_{0} \mathrm{z}_{0}-\mathrm{d}_{0} \mathrm{z}_{0}\left\|\varphi_{2}\right\|^{2}+\dot{\mathrm{x}}_{2 \mathrm{c}}}{\beta}
$$

And the error dynamic becomes

$$
\dot{\mathrm{z}}_{0}=-\mathrm{k}_{0} \mathrm{z}_{0}-\mathrm{d}_{0} \mathrm{z}_{0}\left\|\varphi_{2}\right\|^{2}+\varphi_{2}^{\mathrm{t}} \Delta
$$

\section{b- Control law $u_{1}$}

As mentioned in the previous section, the speed control is done in terms of two steps:

Step 1: Virtual control

Firstly, let's define the error variable $z_{1}$ as: $z_{1}=\eta-\eta_{c}$

According to $(14 \mathrm{e})$, the dynamic equation of the error is:

$$
\dot{\mathrm{z}}_{1}=\hat{\mathrm{g}}_{5}(\eta)+\hat{\Gamma}_{\mathrm{em}}-\dot{\eta}_{\mathrm{C}}+\varphi_{0}^{\mathrm{t}} \Delta
$$


The virtual control $\Gamma_{\mathrm{emc}}$ for the unperturbed system can be determined in the same way as a). Using the Lyapunov candidate function $\mathrm{V}_{01}=\frac{1}{2} \mathrm{z}_{1}^{2}$ and choosing $-\mathrm{k}_{1} \mathrm{z}_{1}=\dot{\mathrm{z}}_{1}$ yields $\hat{\mathrm{g}}_{5}(\eta)+\Gamma_{\mathrm{emc}}-\dot{\eta}_{\mathrm{C}}=-\mathrm{k}_{1} \mathrm{z}_{1}$

For the perturbed system, using nonlinear damping lemma to the system (24), the virtual control $\Gamma_{\mathrm{emc}}$ verifies:

$$
\hat{\mathrm{g}}_{5}(\eta)+\Gamma_{\mathrm{emc}}-\dot{\eta}_{\mathrm{C}}=-\mathrm{k}_{1} \mathrm{z}_{1}-\mathrm{d}_{1} \mathrm{z}_{1}\left\|\varphi_{0}\right\|^{2}
$$

Hence, the virtual control $\Gamma_{\mathrm{emc}}$ is: $\Gamma_{\mathrm{emc}}=-\mathrm{k}_{1} \mathrm{z}_{1}-\mathrm{d}_{1} \mathrm{z}_{1}\left\|\varphi_{0}\right\|^{2}-\hat{\mathrm{g}}_{5}(\eta)+\dot{\eta}_{\mathrm{C}}$

Step 2: control law $\mathrm{u}_{1}$

Secondly, let's consider the error variable $z_{2}$ defined as: $z_{2}=\hat{\Gamma}_{\mathrm{em}}-\Gamma_{\mathrm{emc}}$

We will first express $\dot{z}_{1}$ and $\dot{z}_{2}$. By rewriting (24) as:

$\dot{\mathrm{z}}_{1}=\left(\hat{\Gamma}_{\mathrm{em}}-\Gamma_{\mathrm{emc}}\right)+\left(\hat{\mathrm{g}}_{5}(\eta)+\Gamma_{\mathrm{emc}}-\dot{\eta}_{\mathrm{C}}\right)+\varphi_{0}^{\mathrm{t}} \Delta$

And using (26) and (28), one can make $\dot{z}_{1}$ as:

$\dot{\mathrm{z}}_{1}=\mathrm{z}_{2}-\mathrm{k}_{1} \mathrm{z}_{1}-\mathrm{d}_{1} \mathrm{z}_{1}\left\|\varphi_{0}\right\|^{2}+\varphi_{0}^{\mathrm{t}} \Delta$

For the error $z_{2}$, by replacing $\hat{\Gamma}_{\mathrm{em}}$ and $\Gamma_{\mathrm{emc}}$ according to (14) and (27), the Equation (28) can be rewritten as:

$$
\begin{aligned}
\mathrm{z}_{2} & =\mathrm{a}\left(\mathrm{x}_{1} \hat{\mathrm{x}}_{4}-\mathrm{x}_{2} \hat{\mathrm{x}}_{3}\right)-\dot{\eta}_{\mathrm{C}}+\mathrm{k}_{1} \mathrm{z}_{1}-\mathrm{F}\left(\mathrm{z}_{1}+\eta_{\mathrm{C}}\right)+\hat{\Gamma}+\mathrm{d}_{1} \mathrm{z}_{1}\left(\mathrm{a}^{2} \mathrm{x}_{1}^{2}+\mathrm{a}^{2} \mathrm{x}_{2}^{2}+1\right) \\
& =\mathrm{f}\left(\mathrm{x}_{1}, \mathrm{x}_{2}, \hat{\mathrm{x}}_{3}, \hat{\mathrm{x}}_{4}, \mathrm{z}_{1}, \eta_{\mathrm{C}}, \dot{\eta}_{\mathrm{C}}, \hat{\Gamma}\right)
\end{aligned}
$$

Hence, the derivative $\dot{\mathrm{z}}_{2}$ is:

$$
\dot{\mathrm{z}}_{2}=\frac{\partial \mathrm{f}}{\partial \mathrm{x}_{1}} \dot{\mathrm{x}}_{1}+\frac{\partial \mathrm{f}}{\partial \mathrm{x}_{2}} \dot{\mathrm{x}}_{2}+\frac{\partial \mathrm{f}}{\partial \hat{\mathrm{x}}_{3}} \dot{\hat{\mathrm{x}}}_{3}+\frac{\partial \mathrm{f}}{\partial \hat{\mathrm{x}}_{4}} \dot{\hat{\mathrm{x}}}_{4}+\frac{\partial \mathrm{f}}{\partial \mathrm{z}_{1}} \dot{\mathrm{z}}_{1}+\frac{\partial \mathrm{f}}{\partial \eta_{\mathrm{C}}} \dot{\eta}_{\mathrm{C}}+\frac{\partial \mathrm{f}}{\partial \dot{\eta}_{\mathrm{C}}} \ddot{\eta}_{\mathrm{C}}+\frac{\partial \mathrm{f}}{\partial \hat{\Gamma}} \dot{\hat{\Gamma}}
$$

To update the aerodynamic torque, one proposes the dynamic of the estimation error $\tilde{\Gamma}_{\mathrm{t}}$ as [20]: $\quad \dot{\tilde{\Gamma}}_{\mathrm{t}}=-\lambda_{\mathrm{t}} \tilde{\Gamma}_{\mathrm{t}}+\mathrm{v}_{\mathrm{t}}$

Where, $\lambda_{t}$ is a positive design constant and $v_{t}$ is a term to be determined after, by studying the overall stability of the system.

In the case where the aerodynamic torque is assumed to be constant or slowly varying in time, the Equation (10) allows us to express the derivative $\dot{\hat{\Gamma}}$ as:

$$
\dot{\hat{\Gamma}}=\lambda_{\mathrm{t}} \tilde{\Gamma}_{\mathrm{t}}-\mathrm{v}_{\mathrm{t}}
$$

This equation combined with (3e), by taking $\hat{x}$ instead of $x$, gives the update law $\hat{\Gamma}$ as follows:

$$
\left\{\begin{array}{l}
\dot{\xi}=-\lambda_{t} \xi-\lambda_{t} h(\hat{x})+\lambda_{t}\left(F-\lambda_{t}\right) \eta-v_{t} \\
\hat{\Gamma}_{t}=\xi+\lambda_{t} \eta
\end{array}\right.
$$

Where $\xi$ is an intermediate variable.

Using (14) and (33), $\dot{z}_{2}$ becomes:

$$
\begin{aligned}
\dot{\mathrm{z}}_{2}= & {\left[\left(\beta \frac{\partial \mathrm{f}}{\partial \mathrm{x}_{1}}+\alpha \frac{\partial \mathrm{f}}{\partial \hat{\mathrm{x}}_{3}}\right) \mathrm{u}_{1}+\left(\beta \frac{\partial \mathrm{f}}{\partial \mathrm{x}_{2}}+\alpha \frac{\partial \mathrm{f}}{\partial \hat{\mathrm{x}}_{4}}\right) \mathrm{u}_{2}\right]+\left[\left(\frac{\partial \mathrm{f}}{\partial \mathrm{x}_{1}} \varphi_{1}+\frac{\partial \mathrm{f}}{\partial \mathrm{x}_{2}} \varphi_{2}+\frac{\partial \mathrm{f}}{\partial \mathrm{z}_{1}} \varphi_{0}\right)^{\mathrm{t} \Delta}\right.} \\
& \left.+\left(0,0, \lambda_{\mathrm{t}}\right) \Delta\right]+\left[\frac{\partial \mathrm{f}}{\partial \mathrm{x}_{1}} \mathrm{~g}_{1}(\hat{\mathrm{x}})+\frac{\partial \mathrm{f}}{\partial \mathrm{x}_{2}} \mathrm{~g}_{2}(\hat{\mathrm{x}})+\frac{\partial \mathrm{f}}{\partial \hat{\mathrm{x}}_{3}} \mathrm{~g}_{3}(\hat{\mathrm{x}})+\frac{\partial \mathrm{f}}{\partial \hat{\mathrm{x}}_{4}} \mathrm{~g}_{4}(\hat{\mathrm{x}})+\frac{\partial \mathrm{f}}{\partial \mathrm{z}_{1}}\left(\hat{\mathrm{g}}_{5}(\eta)\right.\right. \\
& \left.\left.+\hat{\Gamma}_{\mathrm{em}}-\dot{\eta}_{\mathrm{C}}\right)+\frac{\partial \mathrm{f}}{\partial \eta_{\mathrm{C}}} \dot{\eta}_{\mathrm{C}}+\frac{\partial \mathrm{f}}{\partial \dot{\eta}_{\mathrm{C}}} \ddot{\eta}_{\mathrm{C}}-\mathrm{v}_{\mathrm{t}}\right]
\end{aligned}
$$

This can be simplified by denoting: 


$$
\begin{aligned}
& \mathrm{f}_{1}=\frac{\partial \mathrm{f}}{\partial \mathrm{x}_{1}}=\mathrm{a} \hat{\mathrm{x}}_{4}+2 \mathrm{~d}_{1} \mathrm{z}_{1} \mathrm{a}^{2} \mathrm{x}_{1} ; \quad \mathrm{f}_{2}=\frac{\partial \mathrm{f}}{\partial \mathrm{x}_{2}}=-\mathrm{a} \hat{\mathrm{x}}_{3}+2 \mathrm{~d}_{1} \mathrm{z}_{1} \mathrm{a}^{2} \mathrm{x}_{2} ; \quad \mathrm{f}_{3}=\frac{\partial \mathrm{f}}{\partial \hat{\mathrm{x}}_{3}}=-\mathrm{ax}_{2} ; \\
& \mathrm{f}_{4}=\frac{\partial \mathrm{f}}{\partial \hat{\mathrm{x}}_{4}}=\mathrm{ax}_{1} ; \mathrm{f}_{5}=\frac{\partial \mathrm{f}}{\partial \eta_{\mathrm{C}}}=-\hat{\mathrm{F}} ; \mathrm{f}_{6}=\frac{\partial \mathrm{f}}{\partial \mathrm{z}_{1}}=\mathrm{k}_{1}-\hat{\mathrm{F}}+\mathrm{d}_{1}\left(\mathrm{a}^{2} \mathrm{x}_{1}^{2}+\mathrm{a}^{2} \mathrm{x}_{2}^{2}+1\right) ; \frac{\partial \mathrm{f}}{\partial \dot{\eta}_{\mathrm{C}}}=-1 ; \\
& \frac{\partial \mathrm{f}}{\partial \hat{\Gamma}}=1 ; \mathrm{a}_{21}=\beta \frac{\partial \mathrm{f}}{\partial \mathrm{x}_{1}}+\alpha \frac{\partial \mathrm{f}}{\partial \hat{\mathrm{x}}_{3}}=\beta \mathrm{f}_{1}+\alpha \mathrm{f}_{1} ; \quad \mathrm{a}_{22}=\beta \frac{\partial \mathrm{f}}{\partial \mathrm{x}_{2}}+\alpha \frac{\partial \mathrm{f}}{\partial \hat{\mathrm{x}}_{4}}=\beta \mathrm{f}_{1}+\alpha \mathrm{f}_{1} \\
& \psi_{21}=\frac{\partial \mathrm{f}}{\partial \mathrm{x}_{1}} \mathrm{~g}_{1}(\hat{\mathrm{x}})+\frac{\partial \mathrm{f}}{\partial \mathrm{x}_{2}} \mathrm{~g}_{2}(\hat{\mathrm{x}})+\frac{\partial \mathrm{f}}{\partial \hat{\mathrm{x}}_{3}} \mathrm{~g}_{3}(\hat{\mathrm{x}})+\frac{\partial \mathrm{f}}{\partial \hat{\mathrm{x}}_{4}} \mathrm{~g}_{4}(\hat{\mathrm{x}})+\frac{\partial \mathrm{f}}{\partial \mathrm{z}_{1}}\left(\hat{\mathrm{g}}_{5}(\eta)+\hat{\Gamma}_{\mathrm{em}}-\dot{\eta}_{\mathrm{C}}\right) \\
& +\frac{\partial \mathrm{f}}{\partial \eta_{\mathrm{C}}} \dot{\eta}_{\mathrm{C}}+\frac{\partial \mathrm{f}}{\partial \dot{\eta}_{\mathrm{C}}} \ddot{\eta}_{\mathrm{C}}-\mathrm{v}_{\mathrm{t}} \\
& =\mathrm{f}_{1} \mathrm{~g}_{1}(\hat{\mathrm{x}})+\mathrm{f}_{2} \mathrm{~g}_{2}(\hat{\mathrm{x}})+\mathrm{f}_{3} \mathrm{~g}_{3}(\hat{\mathrm{x}})+\mathrm{f}_{4} \mathrm{~g}_{4}(\hat{\mathrm{x}})+\mathrm{f}_{5} \dot{\eta}_{\mathrm{C}}+\mathrm{f}_{6}\left(\hat{\mathrm{g}}_{5}(\eta)+\hat{\Gamma}_{\mathrm{em}}-\dot{\eta}_{\mathrm{C}}\right)-\ddot{\eta}_{\mathrm{C}}-\mathrm{v}_{\mathrm{t}} \\
& \left(\psi_{22}\right)^{\mathrm{t}}=\left(\frac{\partial \mathrm{f}}{\partial \mathrm{x}_{1}} \varphi_{1}+\frac{\partial \mathrm{f}}{\partial \mathrm{x}_{2}} \varphi_{2}+\frac{\partial \mathrm{f}}{\partial \mathrm{z}_{1}} \varphi_{0}\right)^{\mathrm{t}}+\left(0,0, \lambda_{\mathrm{t}}\right) \\
& =\left(f_{1} c_{1}-f_{2} n_{1} \eta-f_{6} a x_{2} ; f_{1} n_{1} \eta+f_{2} c_{1}+f_{6} a x_{1} ; f_{6}+\lambda_{t}\right)
\end{aligned}
$$

Hence, $\dot{z}_{2}$ takes the following form:

$$
\dot{\mathrm{z}}_{2}=\mathrm{a}_{21} \mathrm{u}_{1}+\mathrm{a}_{22} \mathrm{u}_{2}+\psi_{21}+\left(\psi_{22}\right)^{\mathrm{t}} \Delta=\vartheta+\left(\psi_{22}\right)^{\mathrm{t}} \Delta
$$

Where $\vartheta$ is an intermediate control variable given by: $\vartheta=a_{21} u_{1}+a_{22} u_{2}+\psi_{21}$

If the $\Delta$-terms were not present in (30) and (35), the derivatives $\dot{\mathrm{z}}_{1}$ and $\dot{\mathrm{z}}_{2}$ would become:

$$
\left\{\begin{array}{l}
\dot{\mathrm{z}}_{1}=\mathrm{z}_{2}-\mathrm{k}_{1} \mathrm{z}_{1}-\mathrm{d}_{1} \mathrm{z}_{1}\left\|\varphi_{0}\right\|^{2} \\
\dot{\mathrm{z}}_{2}=\vartheta
\end{array}\right.
$$

With the augmented Lyapunov function: $V=\frac{1}{2} z_{1}^{2}+\frac{1}{2} z_{2}^{2}$

Whose derivative $\dot{V}$ is written, according to (37), as:

$$
\dot{\mathrm{V}}=-\mathrm{k}_{1} \mathrm{z}_{1}^{2}-\mathrm{d}_{1} \mathrm{z}_{1}^{2}\left\|\varphi_{0}\right\|^{2}+\mathrm{z}_{2}\left(\mathrm{z}_{1}+\vartheta\right)
$$

we can make it negative, by choosing:

$$
\vartheta=-\mathrm{z}_{1}-\mathrm{k}_{2} \mathrm{z}_{2}
$$

To compensate for the presence of $\Delta$-term, the nonlinear damping lemma is used to the system (35) and gives: $\quad \vartheta=-\mathrm{z}_{1}-\mathrm{k}_{2} \mathrm{z}_{2}-\mathrm{d}_{2} \mathrm{z}_{2}\left\|\psi_{22}\right\|^{2}$

Hence, the Equation (36) gives the control law $\mathrm{u}_{1}$ as:

$$
\mathrm{u}_{1}=\frac{-\mathrm{k}_{2} \mathrm{z}_{2}-\mathrm{z}_{1}-\psi_{21}-\mathrm{d}_{2} \mathrm{z}_{2}\left\|\psi_{22}\right\|^{2}-\mathrm{a}_{22} \mathrm{u}_{2}}{\mathrm{a}_{21}}
$$

Using (40), the error dynamic expressed in (35) becomes:

$$
\dot{\mathrm{z}}_{2}=-\mathrm{k}_{2} \mathrm{z}_{2}-\mathrm{z}_{1}-\mathrm{d}_{2} \mathrm{z}_{2}\left\|\psi_{22}\right\|^{2}+\psi_{22}^{\mathrm{t}} \Delta
$$

Remark:

For the unperturbed system, the term $\mathrm{a}_{21}$ takes the following form:

$$
\mathrm{a}_{21}=\beta \mathrm{f}_{1}+\alpha \mathrm{f}_{3}=\mathrm{a}\left(\beta \hat{\mathrm{x}}_{4}-\mathrm{ax}_{2}\right) \approx-\frac{\mathrm{a}}{\sigma \mathrm{L}_{\mathrm{r}} \mathrm{L}_{\mathrm{r}}}\left(\mathrm{L}_{\mathrm{r}} \mathrm{i}_{\mathrm{sq}}+\mathrm{L}_{\mathrm{m}} \mathrm{i}_{\mathrm{rq}}\right)=-\frac{\mathrm{a}}{\sigma \mathrm{L}_{\mathrm{r}} \mathrm{L}_{\mathrm{r}}} \phi_{\mathrm{sq}}
$$

Since the effect of the stator resistance is negligible especially in high power, the flux and the voltage vectors are substantially orthogonal. Then, with the frame used for the induction machine, the term $\left|\phi_{\mathrm{sq}}\right|$ is equal to the amplitude of the stator flux. Consequently, the factor $a_{21}$ becomes different from zero as soon as the system is connected to the grid. 


\section{c- Update laws and overall stability}

One can remark that the control laws $\mathrm{u}_{1}$ and $\mathrm{u}_{2}$ established previously still contain the unknown term $\mathrm{v}_{\mathrm{t}}$. To determine $\mathrm{v}_{\mathrm{t}}$ and show the overall stability of the system, one proposes the following Lyapunov function:

$$
\mathrm{V}=\frac{1}{2} \mathrm{z}_{0}^{2}+\frac{1}{2} \mathrm{z}_{1}^{2}+\frac{1}{2} \mathrm{z}_{2}^{2}+\frac{1}{4 \mathrm{a}_{3}}\left(\frac{1}{\mathrm{~d}_{0}}+\frac{1}{\mathrm{~d}_{1}}+\frac{1}{\mathrm{~d}_{2}}\right) \mathrm{V}_{\text {obs }}+\frac{\tilde{\Gamma}_{\mathrm{t}}^{2}}{2}
$$

So as to simplify calculation of the derivative $\dot{V}$, one denotes:

$$
\begin{aligned}
& \varphi_{0}^{\mathrm{t}}=\left(\mathrm{ax}_{1},-\mathrm{ax}_{2}, 1\right)=\left(\mathrm{w}_{01}, \mathrm{w}_{02}, 1\right) ; \varphi_{2}^{\mathrm{t}}=\left(-\mathrm{n}_{1} \eta, \mathrm{c}_{1}, 0\right)=\left(\mathrm{w}_{21}, \mathrm{w}_{22}, 0\right) ; \\
& \left(\psi_{22}\right)^{\mathrm{t}}=\left(\mathrm{f}_{1} \mathrm{c}_{1}-\mathrm{f}_{2} \mathrm{n}_{1} \eta+\mathrm{f}_{6} \mathrm{ax}_{1} ; \mathrm{f}_{1} \mathrm{n}_{1} \eta+\mathrm{f}_{2} \mathrm{c}_{1}-\mathrm{f}_{6} \mathrm{ax}_{2} ; \mathrm{f}_{6}+\lambda_{\mathrm{t}}\right)=\left(\mathrm{w}_{31}, \mathrm{w}_{32}, \mathrm{w}_{33}\right)
\end{aligned}
$$

As a result, errors $\dot{z}_{0}, \dot{z}_{1}$ and $\dot{z}_{2}$ given in (22), (30) and (42), respectively, become

$$
\left\{\begin{array}{l}
\dot{\mathrm{z}}_{0}=-\mathrm{k}_{0} \mathrm{z}_{0}-\mathrm{d}_{0} \mathrm{z}_{0}\left(\mathrm{w}_{21}^{2}+\mathrm{w}_{22}^{2}\right)+\left(\mathrm{w}_{21} \tilde{\mathrm{x}}_{3}+\mathrm{w}_{22} \tilde{\mathrm{x}}_{4}\right) \\
\dot{\mathrm{z}}_{1}=\mathrm{z}_{2}-\mathrm{k}_{1} \mathrm{z}_{1}-\mathrm{d}_{1} \mathrm{z}_{1}+\widetilde{\Gamma}_{\mathrm{t}}-\mathrm{d}_{1} \mathrm{z}_{1}\left(\mathrm{w}_{01}^{2}+\mathrm{w}_{02}^{2}\right)+\left(\mathrm{w}_{01} \tilde{\mathrm{x}}_{3}+\mathrm{w}_{02} \tilde{\mathrm{x}}_{4}\right) \\
\dot{\mathrm{z}}_{2}=-\mathrm{k}_{2} \mathrm{z}_{2}-\mathrm{z}_{1}-\mathrm{d}_{2} \mathrm{z}_{2} \mathrm{w}_{33}^{2}+\mathrm{w}_{33} \tilde{\Gamma}_{\mathrm{t}}-\mathrm{d}_{2} \mathrm{z}_{2}\left(\mathrm{w}_{31}^{2}+\mathrm{w}_{32}^{2}\right)+\left(\mathrm{w}_{31} \tilde{\mathrm{x}}_{3}+\mathrm{w}_{32} \tilde{\mathrm{x}}_{4}\right)
\end{array}\right.
$$

Using (13b), (32) and (44), the derivative $\dot{V}$ takes the following form:

$$
\begin{aligned}
\dot{V} & =-k_{0} z_{0}^{2}-k_{1} z_{1}^{2}-k_{2} z_{2}^{2}-\lambda_{t} \tilde{\Gamma}_{t}^{2}-d_{1} z_{1}^{2}-d_{2} z_{2}^{2} w_{33}^{2}-\frac{1}{d_{0}}\left(\frac{\tilde{x}_{3}}{2}-d_{0} z_{0} w_{21}\right)^{2}-\frac{1}{d_{0}}\left(\frac{\tilde{x}_{4}}{2}-d_{0} z_{0} w_{22}\right)^{2} \\
& -\frac{1}{d_{1}}\left(\frac{\tilde{x}_{3}}{2}-d_{1} z_{1} w_{01}\right)^{2}-\frac{1}{d_{1}}\left(\frac{\tilde{x}_{4}}{2}-d_{1} z_{1} w_{02}\right)^{2}-\frac{1}{d_{2}}\left(\frac{\tilde{x}_{3}}{2}-d_{2} z_{1} w_{31}\right)^{2}-\frac{1}{d_{2}}\left(\frac{\tilde{x}_{4}}{2}-d_{2} z_{2} w_{32}\right)^{2} \\
& +\left(z_{1}+z_{2} w_{33}+v_{t}\right) \tilde{\Gamma}_{t}
\end{aligned}
$$

To get the unknown term $\mathrm{v}_{\mathrm{t}}$, we can just cancel the factor with $\tilde{\Gamma}_{t}$ on the right side of this expression. Hence, this term is:

$$
\mathrm{v}_{\mathrm{t}}=-\mathrm{z}_{1}-\mathrm{z}_{2} \mathrm{w}_{33}=-\mathrm{z}_{1}-\mathrm{z}_{2}\left(\mathrm{f}_{6}+\lambda_{\mathrm{t}}\right)
$$

With this choice, we can also write:

$$
\dot{\mathrm{V}} \leq-\mathrm{k}_{0} \mathrm{z}_{0}^{2}-\mathrm{k}_{1} \mathrm{z}_{1}^{2}-\mathrm{k}_{2} \mathrm{z}_{2}^{2}-\lambda_{\mathrm{t}} \tilde{\Gamma}_{\mathrm{t}}^{2} \leq 0 \text {. }
$$

Finally, we conclude that errors $\mathrm{z}_{0}, \mathrm{z}_{1}, \mathrm{z}_{2}$ and $\tilde{\Gamma}_{\mathrm{t}}$ converge to zero.

\subsection{GSC Control Design}

We will determine the control laws $\mathrm{v}_{1}$ and $\mathrm{v}_{2}$ necessary for the grid side converter so that the variables $\zeta_{1}, \zeta_{2}$ and $\zeta_{3}$ can track their references denoted $\zeta_{1 \mathrm{c}}, \zeta_{2 \mathrm{c}}$ and $\zeta_{3 \mathrm{c}}$, respectively. With the convergent observer, one can replace currents $\mathrm{x}_{3}$ and $\mathrm{x}_{4}$ by $\hat{x}_{3}$ and $\hat{x}_{4}$, respectively, in the GSC system Equations (6).

Proposition 2: The following control laws:

$$
\mathrm{v}_{1}=\frac{\ddot{\zeta}_{3 \mathrm{c}}-\dot{\alpha}(\hat{\mathrm{x}}, \mathrm{u})-\gamma \mathrm{f}_{1}(\zeta)-\left(\mathrm{p}_{1}+\mathrm{p}_{3}\right) \mathrm{e}_{1}+\mathrm{e}_{3}\left(\mathrm{p}_{3}{ }^{2}-1\right)}{\lambda \gamma} ; \quad \mathrm{v}_{2}=\frac{-\mathrm{p}_{2} \mathrm{e}_{2}-\mathrm{f}_{2}(\zeta)+\dot{\zeta}_{2 \mathrm{c}}}{\lambda}
$$

With $\mathrm{e}_{1}=\gamma \zeta_{1}-\left(\gamma \zeta_{1}\right)_{\mathrm{c}} ; \mathrm{e}_{2}=\zeta_{2}-\zeta_{2 \mathrm{c}} ; \quad \mathrm{e}_{3}=\zeta_{3}-\zeta_{3 \mathrm{c}} ; \quad\left(\gamma \zeta_{1}\right)_{\mathrm{c}}=-\alpha(\hat{\mathrm{x}}, \mathrm{u})-\mathrm{e}_{3} \mathrm{p}_{3}+\dot{\zeta}_{3 \mathrm{c}}$ $p_{1}, p_{2}$ and $p_{3}$ are positives design constants

Ensure the asymptotic stability for the GSC system. 


\section{Proof}

\section{a- Control law $v_{2}$}

Since the current $\zeta_{2}$ is controlled by the variable $\mathrm{v}_{2}$, one first defines the error variable as:

$\mathrm{e}_{2}=\zeta_{2}-\zeta_{2 \mathrm{c}}$

Using (6b), the dynamic equation of this variable error is:

$$
\dot{\mathrm{e}}_{2}=\mathrm{f}_{2}(\zeta)+\lambda \mathrm{v}_{2}-\dot{\zeta}_{2 \mathrm{c}}
$$

By using the Lyapunov function $\mathrm{V}_{2}=\frac{1}{2} \mathrm{e}_{2}^{2}$ and the choice $\dot{\mathrm{e}}_{2}=-\mathrm{p}_{2} \mathrm{e}_{2}$ where $\mathrm{p}_{2}$ is a positive design constant, we can make the derivative $\dot{\mathrm{V}}_{2}$ as: $\dot{\mathrm{V}}_{2}=-\mathrm{p}_{2} \mathrm{e}_{2}^{2} \leq 0$. Hence, the law of $\mathrm{v}_{2}$ is:

$$
\mathrm{v}_{2}=\frac{-\mathrm{p}_{2} \mathrm{e}_{2}-\mathrm{f}_{2}(\zeta)+\dot{\zeta}_{2 \mathrm{c}}}{\lambda}
$$

\section{b- Control law $v_{1}$}

As signalled at the beginning of this subsection, the control variable $\mathrm{v}_{1}$ can be determined in two steps, by applying the backstepping technique to the equations (6c) and (6a).

Step 1: Virtual control $\left(\gamma \zeta_{1}\right)_{\mathrm{c}}$

The first error variable is: $e_{3}=\zeta_{3}-\zeta_{3 c}$

Using (6c), the dynamic equation of the error $e_{3}$ is given by:

$$
\dot{\mathrm{e}}_{3}=\alpha(\hat{\mathrm{x}}, \mathrm{u})+\gamma \zeta_{1}-\dot{\zeta}_{3 \mathrm{c}}
$$

With the Lyapunov function: $V_{3}=\frac{1}{2} e_{3}^{2}$, the choice $\dot{e}_{3}=-p_{3} e_{3}$, where $p_{3}$ is a positive design constant, makes the derivative $\dot{\mathrm{V}}_{3}$ as: $\dot{\mathrm{V}}_{3}=-\mathrm{p}_{3} \mathrm{e}_{3}^{2} \leq 0$. So, the virtual control $\left(\gamma \zeta_{1}\right)_{\mathrm{c}}$ verifies:

$$
\alpha(\hat{\mathrm{x}}, \mathrm{u})+\left(\gamma \zeta_{1}\right)_{\mathrm{c}}-\dot{\zeta}_{3 \mathrm{c}}=-\mathrm{p}_{3} \mathrm{e}_{3}
$$

and it is given by:

$$
\left(\gamma \zeta_{1}\right)_{c}=-\alpha(\hat{x}, \mathrm{u})-\mathrm{p}_{3} \mathrm{e}_{3}+\dot{\zeta}_{3 \mathrm{c}}
$$

Step 2: control low $\mathrm{v}_{1}$

The second error variable is defined as:

$$
\mathrm{e}_{1}=\gamma \zeta_{1}-\left(\gamma \zeta_{1}\right)_{\mathrm{c}}=\gamma \zeta_{1}+\alpha(\hat{\mathrm{x}}, \mathrm{u})+\mathrm{e}_{3} \mathrm{p}_{3}-\dot{\zeta}_{3 \mathrm{c}}
$$

Let's first determine the derivatives $\dot{\mathrm{e}}_{3}$ and $\dot{\mathrm{e}}_{1}$. Using (52) and (53), we can write (50) as:

$$
\dot{\mathrm{e}}_{3}=\alpha(\hat{\mathrm{x}}, \mathrm{u})+\left(\gamma \zeta_{1}\right)_{\mathrm{c}}-\dot{\zeta}_{3 \mathrm{c}}+\left(\gamma \zeta_{1}-\left(\gamma \zeta_{1}\right)_{\mathrm{c}}\right)=-\mathrm{p}_{3} \mathrm{e}_{3}+\mathrm{e}_{1}
$$

To get $\dot{e}_{1}$, we take the derivative of (53). After that, we use (6a) and (54), which gives

$\dot{\mathrm{e}}_{1}=\lambda \gamma \mathrm{v}_{1}+\gamma \mathrm{f}_{1}(\zeta)+\dot{\alpha}(\hat{\mathrm{x}}, \mathrm{u})-\ddot{\zeta}_{3 \mathrm{c}}+\mathrm{p}_{3} \mathrm{e}_{1}-\mathrm{e}_{3} \mathrm{p}_{3}^{2}$

Considering the following global Lyapunov function:

$$
\mathrm{V}=\frac{1}{2} \mathrm{e}_{1}^{2}+\frac{1}{2} \mathrm{e}_{2}^{2}+\frac{1}{2} \mathrm{e}_{3}^{2}
$$

whose derivative is

$$
\dot{\mathrm{V}}=\mathrm{e}_{1} \dot{\mathrm{e}}_{1}+\mathrm{e}_{2}\left(-\mathrm{p}_{2} \mathrm{e}_{2}\right)+\mathrm{e}_{3}\left(-\mathrm{p}_{3} \mathrm{e}_{3}+\mathrm{e}_{1}\right)=-\mathrm{p}_{2} \mathrm{e}_{2}^{2}-\mathrm{p}_{3} \mathrm{e}_{3}^{2}+\mathrm{e}_{1}\left(\dot{\mathrm{e}}_{1}+\mathrm{e}_{3}\right)
$$


The choice: $\dot{\mathrm{e}}_{1}=-\mathrm{e}_{3}-\mathrm{p}_{1} \mathrm{e}_{1}$ ensure the negativity of $\dot{\mathrm{V}}$ since: $\dot{\mathrm{V}}=-\mathrm{p}_{1} \mathrm{e}_{1}^{2}-\mathrm{p}_{2} \mathrm{e}_{2}^{2}-\mathrm{p}_{3} \mathrm{e}_{3}^{2} \leq 0$

Finally, from (55), we can obtain the control law $\mathrm{v}_{1}$ that stabilizes the GSC as:

$$
\mathrm{v}_{1}=\frac{\ddot{\zeta}_{3 \mathrm{c}}-\dot{\alpha}(\hat{\mathrm{x}}, \mathrm{u})-\gamma \mathrm{f}_{1}(\zeta)-\left(\mathrm{p}_{1}+\mathrm{p}_{3}\right) \mathrm{e}_{1}+\mathrm{e}_{3}\left(\mathrm{p}_{3}^{2}-1\right)}{\lambda \gamma}
$$

\section{Simulation Results}

To validate the previous theoretical development and conclude on the effectiveness of our controller, we constructed two Simulink schemes. The first one corresponds to the conventional case with known mechanical torque $\mathrm{Tt}$, given by the Equation (1a). The second one (unknown case) concerns our adaptive Backstepping controller with nonlinear damping. The electro-mechanical characteristics, used in this work, are given in "Appendix 1". In all simulations, constant references values for DC-link voltage, stator and rotor reactive power were considered and fixed respectively at $1200 \mathrm{~V}$ and $0 \mathrm{VAR}$. The design parameters values used are: $\mathrm{k}_{0}=120$; $\mathrm{k}_{1}=80 ; \mathrm{k}_{2}=120 ; \mathrm{d}_{1}=10^{-2} ; \mathrm{d}_{2}=10^{-4} ; \mathrm{d}_{3}=10^{-3} ; \mathrm{p}_{1}=500 ; \mathrm{p}_{2}=100 ; \mathrm{p}_{3}=500$ and $\lambda_{\mathrm{t}}=10$.

Figures 3 show the good tracking performances in the case of the adaptive control when the wind speed varies with time (Figure 3a). Indeed, from "Figure 3b", which represents the speed $\mathrm{N}$ of the rotor and its reference $\mathrm{N} \_$ref, we can see that the two curves are practically identical, which confirms that the wind turbine operates at optimum TSR (see Equation 1b). To have a unity power factor, the references of $i_{\mathrm{sq}}$ $\left(\mathrm{x}_{2}\right)$ and $\mathrm{i}_{\mathrm{oq}}\left(\zeta_{2}\right)$ were fixed at zero (see Equations (7) and $(9)$ ). "Figures $3 \mathrm{c}$ and $3 \mathrm{~d}$ " show the good current tracking performances for the controller. The same remark can be made regarding the DC-links voltage shown in "Figure $3 \mathrm{e}$ ". "Figure $3 \mathrm{f}$ " that represents variations in time of the mechanical torque and its estimated value confirms the validity of the proposed update law. Also, the simulation shows, through "Figures $3 \mathrm{~g}$ and $3 \mathrm{~h}$ ", that the observed currents reach quickly the measured ones, which confirms the effectiveness of the implemented observer. "Figures $3 \mathrm{i}$ and $3 \mathrm{j}$ " allow us to verify the two operating modes of the generator: According to "Figure $3 \mathrm{~b}$ ", one can remark that, around time $\mathrm{t}=102 \mathrm{~s}$ approximately, the induction machine passes from sub-synchronous operation mode (for $\mathrm{N}<1500 \mathrm{rpm}$ ) to supersynchronous one (for $\mathrm{N}>1500 \mathrm{rpm}$ ). For the stator, "Figure 3i" shows that, for the two operating modes, the grid voltage and the current are in phase. Thus, the active power is always transmitted from the stator to grid regardless of the operation mode. However in "Figure 3j", we can see that the grid voltage and the filter current are in phase opposition for $\mathrm{N}<1500 \mathrm{rpm}$ (for $\mathrm{t}<102.05 \mathrm{~s}$ ) and in phase for $\mathrm{N}>1500 \mathrm{rpm}$ (for $\mathrm{t}>102.05 \mathrm{~s}$ ). Thus, the active power is transmitted from the grid to rotor for subsynchronous operation mode and from the grid to rotor for super-synchronous operation mode. 


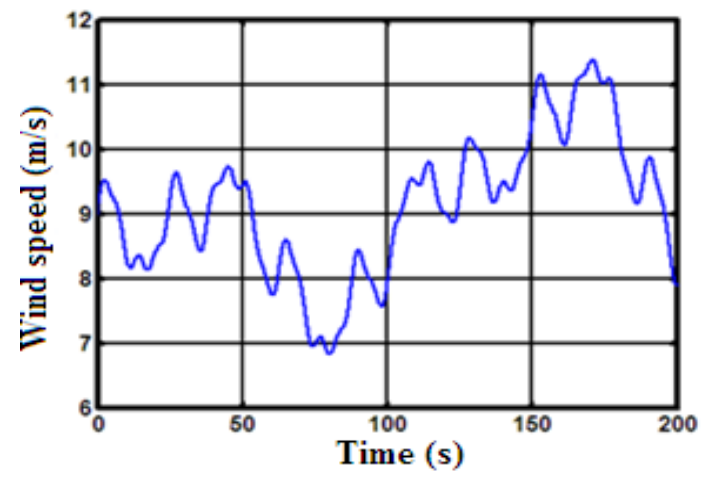

Figure 3a. Time-Varying Wind Speed

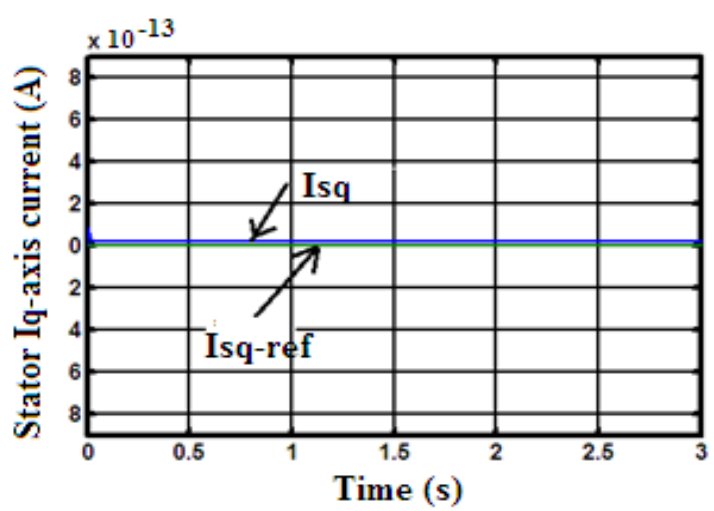

Figure 3c. Stator Q-Axis Current and Its Reference

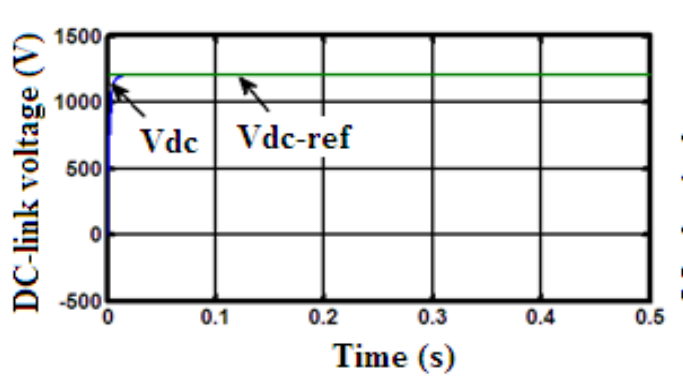

Figure 3e. DC-Link Voltage and Its Reference

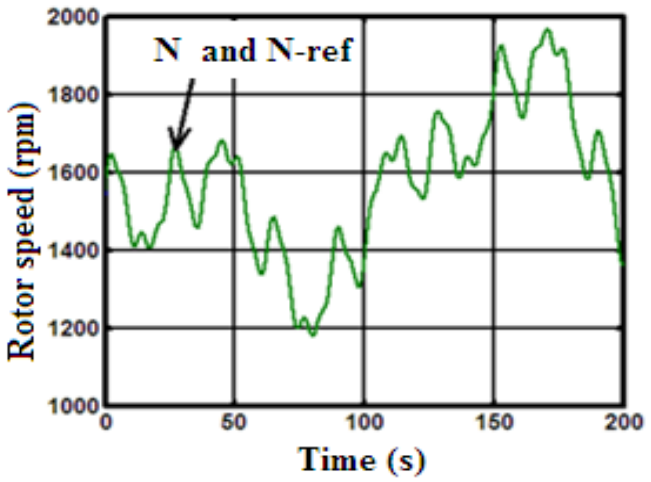

Figure 3b. Rotor Speed and Its Reference

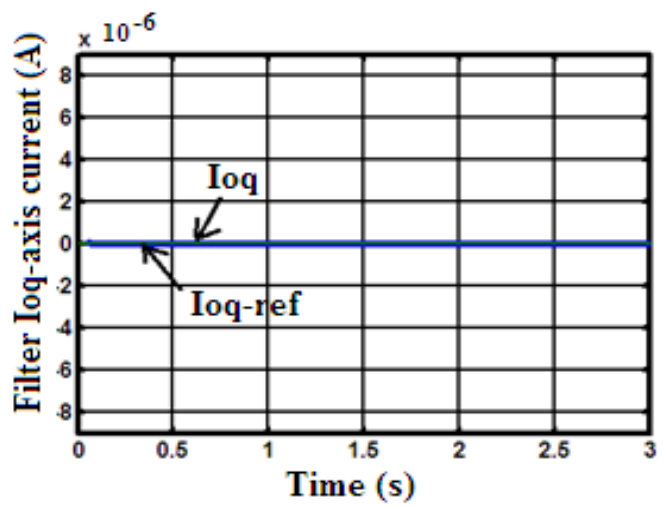

Figure 3d. Filter Q-Axis Current and Its Reference

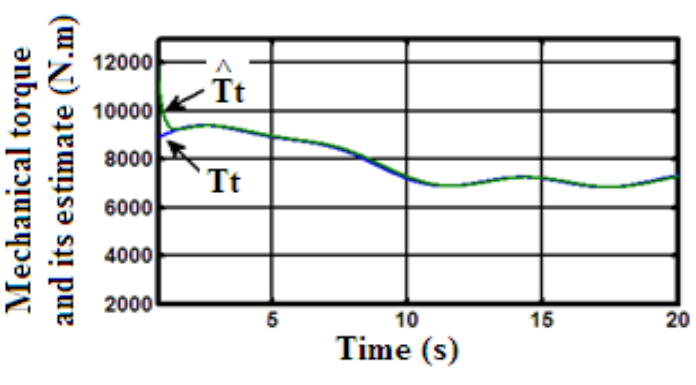

Figure 3f. Mechanical Torque and Its Estimated Value 


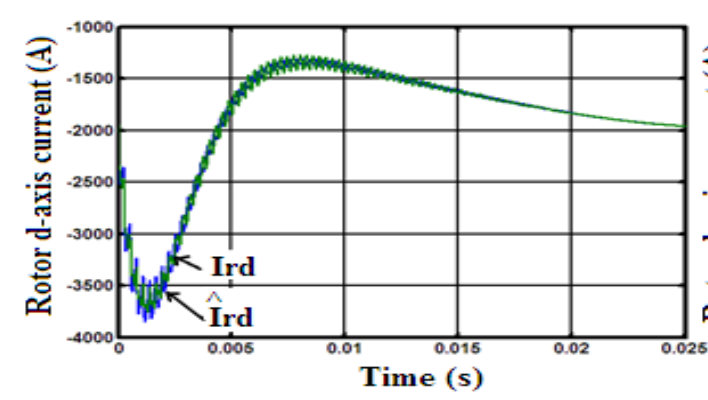

Figure $3 g$. Measured and Observed Current Ird

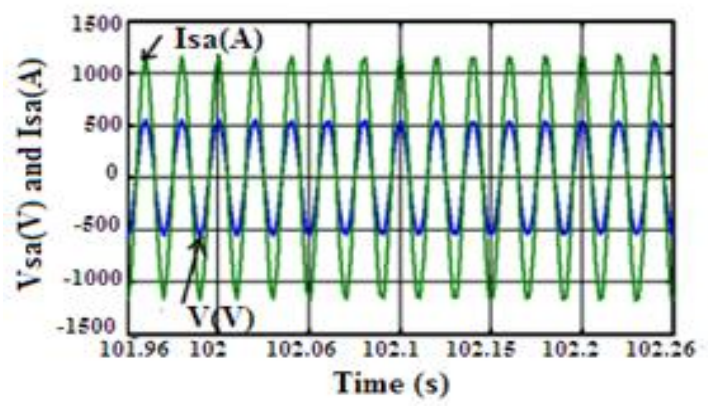

Figure 3i. Stator Isa and Vsa Phase A

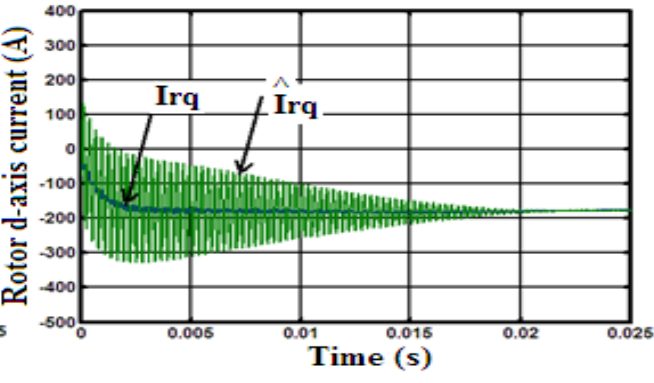

Figure 3h. Measured and Observed Current Irq

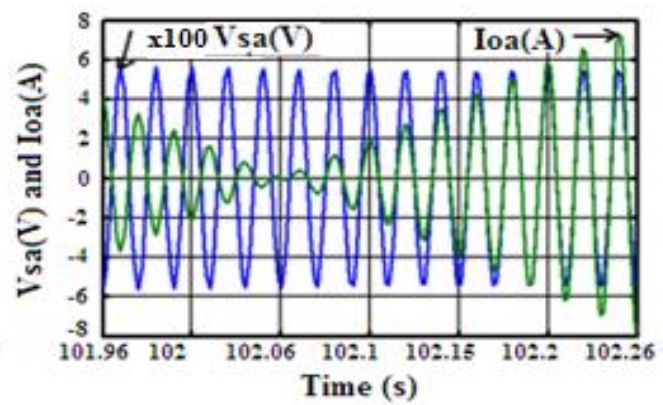

Figure 3j. Filter Current loa and Vsa Phase A

Figure 3. Tracking Capability of the Controller for Time-Varying Wind Speed

In Figure 4, we can see clearly the beneficial effect of the nonlinear damping terms that we have implemented. Indeed, "Figure 4a" gives the speed transient response for a controller with nonlinear damping while "Figure $4 b$ " shows the behavior in the case of $\mathrm{d}_{0}=\mathrm{d}_{1}=\mathrm{d}_{2}=0$. One concludes that this tool improves the transient performance.

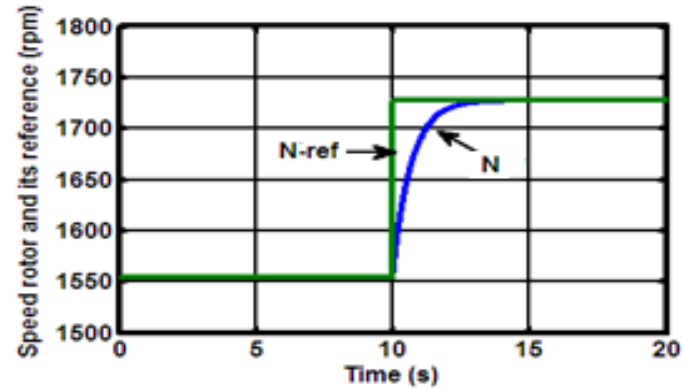

Figure 4a. Tracking with Nonlinear Damping

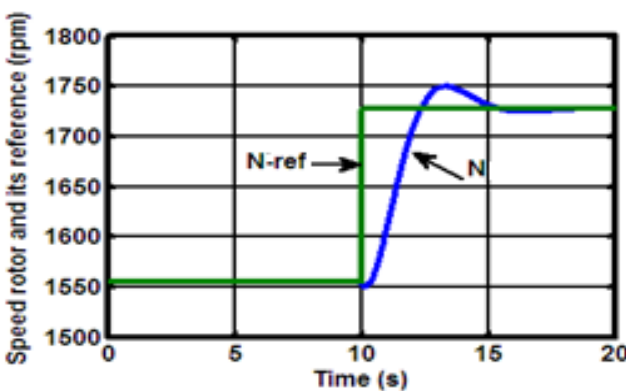

Figure 4b. Tracking without Nonlinear Damping $\left(d_{0}=0 ; d_{1}=0\right.$; $d_{2}=0$ )

Figure 4. Speed Tracking With and Without Nonlinear Damping 
Finally, "Figure 5" illustrates a comparison, in terms of speed tracking performances, between the conventional and adaptive controllers in the presence of perturbations in the mechanical torque $\mathrm{T}_{\mathrm{t}}$. The simulation produces changes, only in the model of the system, at $20 \mathrm{~s}(\Delta \mathrm{Tt}=50 \%)$, at $40 \mathrm{~s}(\Delta \mathrm{Tt}=100 \%)$, at $60 \mathrm{~s}(\Delta \mathrm{Tt}=150 \%)$ and at $80 \mathrm{~s}(\Delta \mathrm{Tt}=200 \%)$. Unlike the conventional Backstepping controller ("Figure $5 b$ "), the adaptive one tracks the constant speed reference despite the changes ("Figure 5a"). This confirms the robustness of the proposed controller.

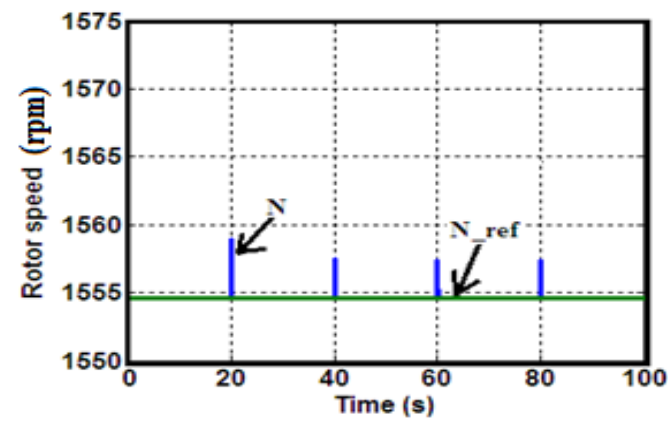

Figure 5a. Tracking for Adaptive Controller

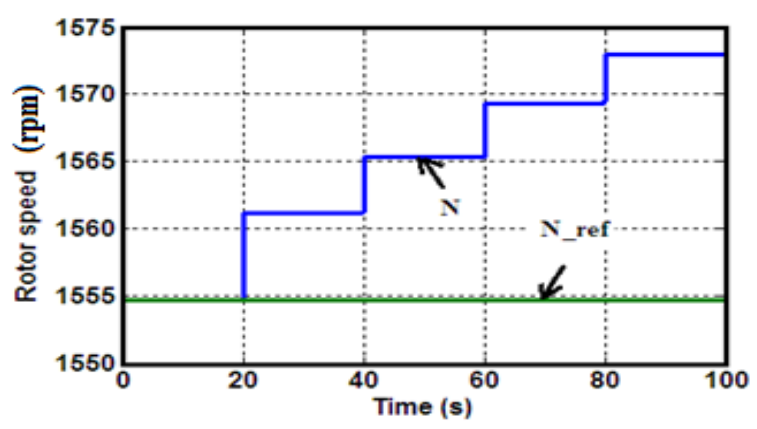

Figure 5b. Tracking without Adaptation

Figure 5. Comparing Speed Regulation Performance of Controllers With and Without Adaptation

\section{Conclusion}

The simulation results presented in this paper show the effectiveness and robustness of the proposed controller for variable-speed DFIG system with unknown rotor currents and mechanical torque. The comparison with the conventional controller also shows the superiority of our adaptive controller in the presence of perturbations in the mechanical torque. On the other hand, the proposed control law guarantees global uniform boundedness and convergence of the solution despite the unknown nonlinear disturbances. The transient results also show that the nonlinear damping yields good speed tracking performance. To improve the performance of the proposed control, further studies could explore in depth the effect of system parameters that depend on temperature like stator and rotor windings resistance.

\section{Appendix 1: Characteristics and Parameters}

\begin{tabular}{|ll|ll|}
\hline Induction Generator: & & Blade Radius & $\mathrm{R}_{\mathrm{t}}=45 \mathrm{~m}$ \\
Rated power & $\mathrm{P}=3 \mathrm{MW}$ & Power coefficient & $\mathrm{C}_{\mathrm{pmax}}=$ \\
Rated stator voltage & $\mathrm{U}=690 \mathrm{~V}$ & 0.48 & \\
Nominal frequency & $\mathrm{fr}=50 \mathrm{~Hz}$ & Optimal TSR & $\lambda_{\mathrm{opt}}=8.14$ \\
Number of pole pairs & $\mathrm{p}=2$ & Mechanical speed multiplier & $\mathrm{G}=100$ \\
Rotor resistance & $\mathrm{R}_{\mathrm{s}}=2.97 \mathrm{e}-3 \Omega$ & $c_{1}=0.5176, c_{2}=116, c_{3}=0.4, c_{4}=5$, \\
Stator resistance & $\mathrm{R}_{\mathrm{r}}=3.82 \mathrm{e}-3 \Omega$ & $c_{5}=21$ and $c_{6}=0.0068$ & \\
Stator inductance & $\mathrm{L}_{\mathrm{s}}=0.012 \mathrm{H}$ & Generator and Turbine: & \\
Rotor inductance & $\mathrm{L}_{\mathrm{r}}=0.0122 \mathrm{H}$ & Moment of inertia & \\
Mutual inductance & $\mathrm{L}_{\mathrm{m}}=12.12 \mathrm{e}-3 \mathrm{H}$ & $\mathrm{J}=254 \mathrm{Kg} \cdot \mathrm{m}^{2}$ & $\mathrm{~F}=0.24$ \\
Wind Turbine: & & Damping coefficient & \\
& & Bus DC- Filter RL: C $=38 \mathrm{mF}, \mathrm{v}_{\mathrm{dc}}=1200$ \\
& & V, R $=0,075 \Omega, \mathrm{L}=0,75 \mathrm{mH}$ & \\
& & &
\end{tabular}




\section{Appendix 2: Nonlinear Damping Lemma}

Consider the system

$\dot{x}=f(x)+g(x) u, \quad f(0)=0$,

Where $\mathrm{u} \in \mathbb{R}$ is the control input and $\mathrm{x} \in \mathbb{R}^{\mathrm{n}}$ is the state. Suppose that there exists a continuously differentiable feedback control law $\mathrm{u}=\alpha(\mathrm{x}), \quad \alpha(0)=0$,

And a smooth, positive definite, radially unbounded function $V: \mathbb{I R}^{\mathrm{n}} \rightarrow \mathrm{IR}_{\mathrm{R}}$ such that:

$\dot{\mathrm{V}}(\mathrm{x}) \leq \mathrm{W}(\mathrm{x}) \leq 0 \quad \forall \mathrm{x} \in \mathrm{IR}^{\mathrm{n}}$ Where $\mathrm{W}: \mathrm{IR}^{\mathrm{n}} \rightarrow \mathrm{IR}$ is positive definite and radially unbounded.

Now consider the perturbed system $\dot{\mathrm{x}}=\mathrm{f}(\mathrm{x})+\mathrm{g}(\mathrm{x})\left[\mathrm{u}+\varphi^{\mathrm{t}}(\mathrm{x}) \Delta(\mathrm{x}, \mathrm{u}, \mathrm{t})\right]$

where $\Delta(\mathrm{x}, \mathrm{u}, \mathrm{t})$ is a $(\mathrm{p} \times 1)$ vector of uncertain nonlinearities which are uniformly bounded for all values of $\mathrm{x}, \mathrm{u}, \mathrm{t}$ and $\varphi(\mathrm{x})$ is a ( $\mathrm{p} \mathrm{x}$ 1) vector for known smooth functions.

For this system, the feedback control $u=\alpha(x)-\kappa \frac{\partial V}{\partial x}(x) g(x)\|\varphi(x)\|^{2}$

guarantees global uniform boundedness and convergence of $\mathrm{x}(\mathrm{t})$.

\|\| is defined as: $\|\mathrm{Y}\|=\sqrt{\mathrm{y}_{1}^{2}+\mathrm{y}_{2}^{2}+\cdots+\mathrm{y}_{\mathrm{n}}^{2}}$ where $\mathrm{Y}=\left(\mathrm{y}_{1}, \mathrm{y}_{2} \cdots, \mathrm{y}_{\mathrm{n}}\right)^{\mathrm{t}}$.

$\kappa \frac{\partial \mathrm{V}}{\partial \mathrm{x}}(\mathrm{x}) \mathrm{g}(\mathrm{x})\|\varphi(\mathrm{x})\|^{2}$ is a nonlinear damping term.

Proof: See [18, section 2.5]

\section{References}

[1] A. Petersson, "Analysis, modeling and control of doubly fed induction generators for wind turbines", PhD. thesis, Champers University of Technology, Sweden, (2005).

[2] R. Datta and V. T. Ranganathan, "Variable-Speed Wind Power Generation Using Doubly Fed Wound Rotor Induction Machine - A Comparison With Alternative Schemes", IEEE Transactions on Energy Conversion, vol. 17, no. 3, (2002), pp. 414-421.

[3] H. Li and Z. Chen, "Overview of different wind generator systems and their comparisons", IET Renewable Power Generation, vol. 2, no. 2, (2008), pp. 123-138.

[4] B. Multon, X. Roboam, B Dakyo, C. Nichita, O Gergaud and H. Ben Ahmed, "Aérogénérateurs électriques", Techniques de l'Ingénieur, Traités de génie électrique, D3960, (2004).

[5] E. Koutroulis and K. Kalaitzakis, "Design of a Maximum Power Tracking System for Wind-EnergyConversion Applications", IEEE Transactions on Industrial Electronics, vol. 53, no. 2, (2006).

[6] L. L. Freris, "Wind Energy Conversion Systems", Englewood Cliffs, NJ: Prentice-Hall, (1990), pp. 182 184.

[7] Y. Hong, S. Lu and C. Chiou, "MPPT for PM wind generator using gradient approximation", Energy Conversion and Management, vol. 50, no. 1, (2009), pp. 82-89.

[8] B. Hopfensperger, D. J. Atkinson and R. Lakin, "Stator-flux-oriented control of a doubly-fed induction machine: with and without position encoder", IEE Proc. Electr. Power Applications, vol. 147 , no. 4, (2000), pp. 241-250.

[9] R. Pena, J. C. Clare and G. M. Asher, "Doubly fed induction generator using back-to-back PWM converters and its application to variable -speed wind-energy generation", IEE Proc. Electr. Power Appl., vol. 143, no. 3, (1996), pp. 231-241.

[10] Y. Zhou, P. Bauer, J.A. Ferreira and J. Pierik, "Control of DFIG Under Unsymmetrical Voltage Dip", IEEE Power Electronics Specialists Conference, (2007), pp.933-938.

[11] S. Peresada, A. Tilli and A. Tonielli, "Robust active-reactive power control of a doubly-fed induction generator”, In Proc. IEEE-IECON', Aachen, Germany, (1998), pp. 1621-1625.

[12] I. Takahashi and T. Noguchi, "A new quick-response and high-efficiency control strategy of an induction motor", IEEE Trans. Ind. Appl., vol. IA-22, no. 5, (1986), pp. 820-827.

[13] T. Noguchi, H. Tomiki, S. Kondo and I. Takahashi, "Direct power control of PWM converter without power-source voltage sensors", IEEE Trans. Ind. Applications, vol. 34, May/June (1998), pp. 473-479.

[14] A. Karthikeyan, S.K. Kummara, C. Nagamani and G. Saravana Ilango, "Power control of grid connected Doubly Fed Induction Generator using Adaptive Back Stepping approach", in Proc 10th IEEE International Conference on Environment and Electrical Engineering EEEIC-2011, Rome, (2011).

[15] S. Heier, "Grid Integration of Wind Energy Conversion Systems", John Wiley \& Sons Ltd, ISBN 0-471-97143-X, (1998).

[16] J.P. Caron, J.P. Hautier, "Modélisation et Commande de la Machine Asynchrone", Edition Technip, Paris, (1995). 
[17] H. Tan and J. Chang, "Field orientation and adaptive backstepping for induction motor control", IEEE Proc., (1999).

[18] M. Krstic, I. Kanellakopoulos and P. Kokotovic, "Nonlinear and adaptive control design", John Wiley \& Sons, Inc., New York, (1995).

[19] I. Kanellakopoulos, P.V. Kokotovic and A.S. Morse, "Systematic design of adaptive controller for feedback linearizable systems", IEEE Trans. Auto Control, vol. 36, no. 11, (1991), pp. 12411253.

[20] Marino, Riccardo, Tomei, Patrizio, Verrelli and M. Cristiano, "Induction Motor Control Design”, Springer-Verlag London, (2010).

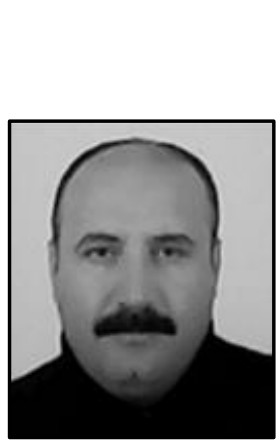

Authors

Mohammed Rachidi, he was born in Boujaâd, Morocco. He received the engineer's degree from Ecole Mohammadia d'Ingénieurs (EMI-Rabat), Morocco, in 1995 and the Master degree from Université Mohammed V, Rabat, Morocco, in 2002. His search interested power electronics and control of electrical machines. Since 1997, he has been working at Ecole Nationale Supérieure d'Arts et Métiers (ENSAM-Meknès), Moulay Ismaïl University, Meknès, Morocco, where he is a Professor in the Department of Electromechanical Engineering.

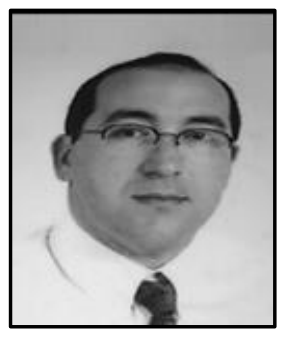

Badr Bououlid Idrissi, he was born in Marrakech, Morocco. $\mathrm{He}$ received the Ph.D. degree from Faculté Polytechnique de Mons, Mons, Belgium, in 1997 and the engineer's degree from Ecole Nationale de l'Industrie Minérale (ENIM-Rabat), Morocco, in 1992. Since 1999, he has been working at Ecole Nationale Supérieure d'Arts et Métiers (ENSAM-Meknès), Moulay Ismaïl University, Meknès, Morocco, where he is a Professor in the Department of Electromechanical Engineering, in the areas of power electronics and electrical machines. 
International Journal of Control and Automation

Vol. 10, No. 2 (2017) 